\title{
ROLE OF ADDITION OF NON-CONTRAST MRI SCAN BRAIN WITH SPECIFIC SEQUENCES (GRE, SWI, TOF ANGIOGRAM \& TOF VENOGRAM SEQUENCES) TO ROUTINE NON-CONTRAST CT SCAN BRAIN AT INITIAL EMERGENCY WORK UP IN EVALUATION OF INTRA CEREBRAL HAEMORRHAGE
}

\author{
Somasekhar R', Praveen Elaprolu ${ }^{2}$
}

${ }^{1}$ Assistant Professor, Department of Radio-diagnosis, Shri Satya Sai Medical College \& Research Institute, Kancheepruam, Tamilnadu. ${ }^{2}$ Senior Resident, Department of Radio-diagnosis, Siddhartha Medical College and Government Hospital, Vijayawada, Andhra Pradesh.

\section{BACKGROUND}

ABSTRACT

The aim of the study is to assess the outcome of addition of non-contrast MRI scan brain with specific sequences (GRE, SWI, TOF Angiogram \& TOF Venogram sequences) when compared to plain non-contrast CT scan brain at initial emergency work up in evaluation of intracerebral haemorrhage. Intraventricular haemorrhage, traumatic subarachnoid haemorrhage, diffuse axonal injury and haemorrhagic contusions. Causes of non-traumatic haemorrhage include hypertension, ruptured aneurysms, arteriovenous malformation (AVM), amyloid angiopathy, haemorrhagic transformation of ischemic infarction, venous haemorrhagic infarct secondary to cortical venous sinus thrombosis.

The aim of the study is to assess the outcome of addition of non-contrast MRI scan brain with specific sequences (GRE, SWI, TOF Angiogram \& TOF Venogram sequences) when compared to plain non-contrast CT scan brain at initial emergency work up in evaluation of intracerebral haemorrhage.

\section{MATERIALS AND METHODS}

This descriptive study spanned a course of 16 months (September 2016 to December 2017) which includes 90 patients with intracerebral haemorrhage on plain CT scan. These patients are subjected to MRI scan immediately as a part of emergency work up and the findings were recorded.

\section{RESULTS}

Out of 90 patients of our study 45 cases (50\%) were traumatic haemorrhage, 18 cases (20\%) were hypertensive haemorrhage, 15 were haemorrhage secondary to aneurysms (16.7\%) and 4 cases due to arteriovenous malformations (4.4\%) remaining 8 cases (8.9\%) include haemorrhage secondary to amyloid angiopathy (3 cases), haemorrhagic metastasis (1 case) \& haemorrhagic transformation of ischemic infarct (4 cases). Among 15 cases of haemorrhage secondary to aneurysms, the aneurysm is identified on MR TOF Angiogram sequence in 12 cases which is not evident on plain CT scan, in rest of the 3 cases the aneurysm is identified on further investigation with CT Angiogram. Among 4 cases of arteriovenous malformations, focal haemorrhage alone seen on CT scan which on MR show underlying nidus \& AV malformation. Among 3 cases of amyloid angiopathy, the larger bleeds are seen on CT scan \& micro-haemorrhages are identified only on MR scan thus aiding in diagnosis \& management. No differences observed between MR and CT scan findings in cases of haemorrhagic metastasis (1 case) \& haemorrhagic transformation of ischemic infarct (4 cases). Among 45 cases of traumatic haemorrhage 15 cases are categorized as diffuse axonal injury based on petechial haemorrhages at grey white matter junction which are appreciated on MR alone \& not evident on CT scan. However, CT scan identified bony skull injuries better than MR scan. Among 18 cases of hypertensive haemorrhage no differences observed between MR and CT scan findings in 15 cases; in rest of the 3 cases, additional micro haemorrhages seen in pons \& cerebellar hemispheres, however no significant alteration in diagnosis or management made. The percentage of subjects with diagnosis made by adding MRI to routine CT scan is calculated based on the data. Among total 90 patients, in 31 cases addition of MR resulted in obtaining the diagnosis. 3 cases are detected on further investigation (CT Angiogram) which were not identified on Plain CT \& MR. In rest of the 56 patients, Plain CT scan alone proved enough for diagnosis as MR did not add any further findings. Thus in $34.4 \%$ of the subjects, diagnosis is made after addition of MRI to routine CT scan.

\section{CONCLUSION}

In cases of intracerebral haemorrhage, MRI added crucial information to CT scan and aided in identifying the cause of intracerebral haemorrhage, diagnosis and management.

\section{KEYWORDS}

Intra Cerebral Haemorrhage, Computed Tomography, Magnetic Resonance Imaging.

HOW TO CITE THIS ARTICLE: Somasekhar R, Elaprolu P. Role of addition of non-contrast MRI scan brain with specific sequences (GRE, SWI, TOF angiogram \& TOF venogram sequences) to routine non-contrast CT scan brain at initial emergency work up in evaluation of intra cerebral haemorrhage. J. Evolution Med. Dent. Sci. 2018;7(16):1999-2002, DOI: 10.14260/jemds/2018/449

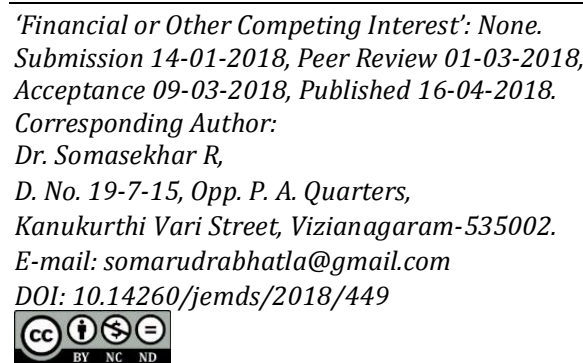

\section{BACKGROUND}

Intracerebral haemorrhage can be Traumatic and Nontraumatic. Traumatic Intracerebral haemorrhage includes patterns of Subdural haemorrhage, Epidural haemorrhage, Intraventricular haemorrhage, traumatic subarachnoid haemorrhage, diffuse axonal injury and haemorrhagic contusions. Causes of non-traumatic haemorrhage include hypertension, ruptured aneurysms, Arteriovenous malformation (AVM), Amyloid angiopathy, haemorrhagic 
transformation of ischemic infarction, venous haemorrhagic infarct secondary to cortical venous sinus thrombosis.

Hypertensive Intra cerebral haemorrhage occurs due to spontaneous rupture of a penetrating arteries deep in the brain parenchyma. Common sites of hypertensive Intra cerebral haemorrhage include basal ganglia, cerebellum and pons.

Cerebral amyloid angiography (CAA) results from amyloid-B peptide deposition within the walls of cerebral arteries, and this deposition results in weakening of arterial wall that may cause cerebral micro haemorrhages, sulcal Sub arachnoid haemorrhage, or larger cerebral Intra parenchymal haemorrhages. Intra cerebral haemorrhage secondary to cerebral amyloid angiopathy is often distinguished from haemorrhage due to hypertension by several imaging characteristics. IPH due to CAA is typically centered in the sub cortical white matter and typically spares the posterior fossa, basal ganglia, and brainstem. ${ }^{1}$

Cerebral aneurysms are focal outpouchings from arteries coursing over the surface of the brain parenchyma. These outpouchings are weakened areas of the arterial wall and are prone to rupture. SAH due to cerebral aneurysm rupture involves the basal cisterns, where the majority of cerebral aneurysm are located, and often extends diffusely throughout the subarachnoid space. The SAH may also extend into the ventricles and brain parenchyma itself depending upon the volume of haemorrhage and location of the ruptured aneurysm. The vast majority of cerebral aneurysms present clinically as sub arachnoid haemorrhage. ${ }^{2}$

Coming to haemorrhagic conversion of ischemic infarction, the Ischemic infraction occurs secondary to thrombotic or thromboembolic blockage of a cerebral artery. The risk of haemorrhagic conversion of an ischemic infarction is increased following intravenous or trans-arterial vessel recanalization. ${ }^{3}$ Cerebral AVMs are characterized by abnormal arteriovenous shunting between cerebral arteries and veins through multiple small channels termed Nidus.

Intra cerebral haemorrhage secondary to venous thrombosis typically does not follow an arterial distribution and is located near the grey-white matter junction. Dural venous sinus thrombosis is more common than cortical vein thrombosis and the dural sinuses may become occluded secondary to skull base infections, hyper coagulable states, dehydration and compression from meningiomas or other duraltumours. 4

\section{Objective \& Study Design}

The aim of the study is to assess the outcome of addition of non-contrast MRI scan brain with specific sequences (GRE, SWI, TOF Angiogram \& TOF Venogram sequences) when compared to plain non-contrast CT scan brain at initial emergency work up in evaluation of Intra cerebral haemorrhage.

\section{Study Design}

It is a descriptive study conducted from August 2016 to November 2017. 208 patients presenting with symptoms of acute cerebral event to our hospital underwent non-contrast CT scan brain. Cases with normal findings \& cases with infarcts were excluded. Inclusion criteria include the cases with intra cerebral haemorrhage. non-contrast MRI scan brain with specific sequences (GRE, SWI, TOF Angiogram \& TOF Venogram sequences) done at this initial emergency work up in the patients included in the study. The findings after CT scan alone, after CT Scan \& MRI are recorded \& compared. Thus, the outcome of addition of MRI to CT scan in initial emergency work up is assessed. All the patients underwent CT Angiogram after stabilization.

\section{MATERIALS AND METHODS}

This descriptive study was carried out in Sri Satya Sai Medical College \& Research Institute \& Spanned a course of 16 months (August 2016 to November 2017) which include 90 patients with Intra cerebral haemorrhage on plain CT scan. These patients are subjected to MRI scan immediately as a part of emergency work up and the findings were recorded. The patients were scanned using GE 16 SLICE CT from base of skull to sella (2.0 mm sections) and above sella $(5.0 \mathrm{~mm}$ sections). MRI scan is done using PHILLIPS 1.5 T ACHIEVA. Axial, coronal and sagittal planes including GRE, SWI and TOF Angiogram \& TOF venogram images were taken.

\section{RESULTS}

Out of 90 patients of our study 45 cases (50\%) were traumatic haemorrhage, 18 cases $(20 \%)$ were hypertensive haemorrhage, 15 were haemorrhage secondary to aneurysms (16.7\%) and 4 cases due to Arteriovenous malformations (4.4\%) remaining 8 cases $(8.9 \%)$ include haemorrhage secondary to amyloid angiopathy (3 cases), haemorrhagic metastasis(1 case) \& haemorrhagic Transformation of ischemic infarct (4 cases).

\begin{tabular}{|c|c|}
\hline Trauma related ICH & 45 cases $(50 \%)$ \\
\hline Hypertensive ICH & 18 cases $(20 \%)$ \\
\hline Aneurysm \& AVM related ICH & 19 cases $(21.1 \%)$ \\
\hline $\begin{array}{c}\text { Others like Amyloid angiopathy, } \\
\text { haemorrhagic metastasis\& haemorrhagic } \\
\text { Transformation of ischemic infarct }\end{array}$ & 8 cases $(8.9 \%)$ \\
\hline Total Cases & $\mathbf{9 0}$ cases \\
\hline
\end{tabular}

Among 15 cases of haemorrhage secondary to aneurysms, the aneurysm is identified on MR TOF Angiogram sequence in 12 cases which is not evident on plain CT scan, in rest of the 3 cases the aneurysm is identified on further investigation with CT Angiogram. Among 4 cases of arteriovenous malformations, focal haemorrhage alone seen on CT scan which on MR show underlying Nidus\& AV Malformation.

Among 3 cases of amyloid angiopathy, the larger bleeds are seen on CT scan \& micro haemorrhages are identified only on MR scan thus aiding in diagnosis \& management.

No differences observed between MR and CT scan findings in cases of haemorrhagic metastasis ( 1 case) \& haemorrhagic Transformation of ischemic infarct (4 cases).

Among 45 cases of traumatic haemorrhage, 15 cases are categorized as diffuse axonal injury based on petechial haemorrhages at grey white matter junction which are appreciated on MR alone \& not evident on CT scan. However, CT scan identified bony skull injuries better than MR scan.

Among 18 cases of hypertensive haemorrhage no differences observed between MR and CT scan findings in 15 cases, in rest of the 3 cases additional micro haemorrhages seen in pons \& cerebellar hemispheres, however no significant alteration in diagnosis or management made. 


\section{Analysis}

The percentage of subjects with diagnosis made by adding MRI to routine CT scan is calculated based on the data Among total 90 patients, in 31 cases addition of MR resulted in obtaining the diagnosis. 3 cases are detected on further investigation (CT Angiogram) which were not identified on Plain CT \& MR. In rest of the 56 patients Plain CT scan alone proved enough for diagnosis as MR did not add any further findings. Thus in $34.4 \%$ of the subjects, diagnosis is made after addition of MRI to routine CT scan.

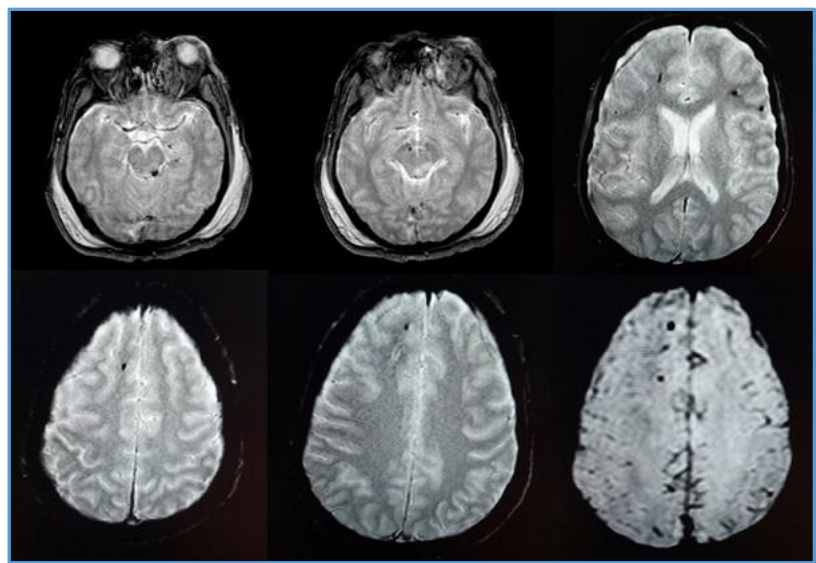

Figure 1. GRE \& SWI Sequences show micro haemorrhages at bilateral frontal \& left parietal grey white matter junction. Haemorrhagic \& non-haemorrhagic contusions seen in brain stem--- Case of Diffuse Axonal Injury

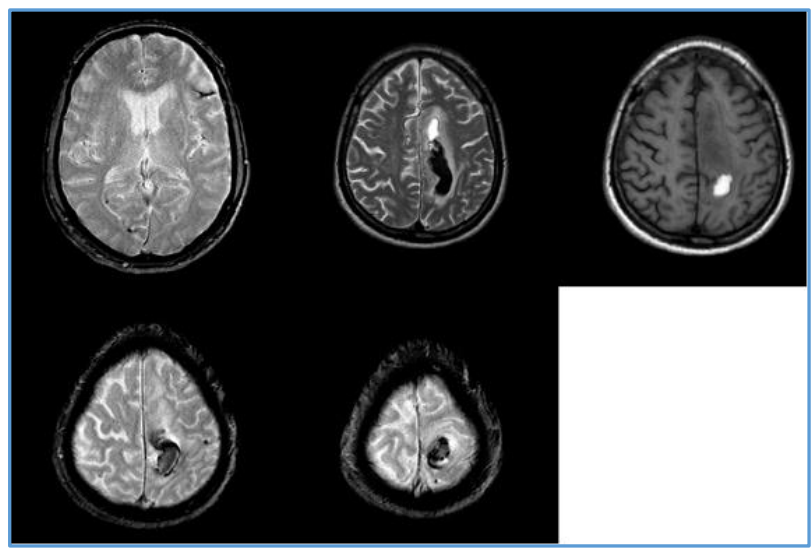

Figure 2. GRE, T2 and T1 MR sequences show large intra cerebral haemorrhage in left centrum semiovale, subcortical micro haemorrhages, and focal sub arachnoid haemorrhage in left frontal sulci--case of amyloid cerebral angiopathy

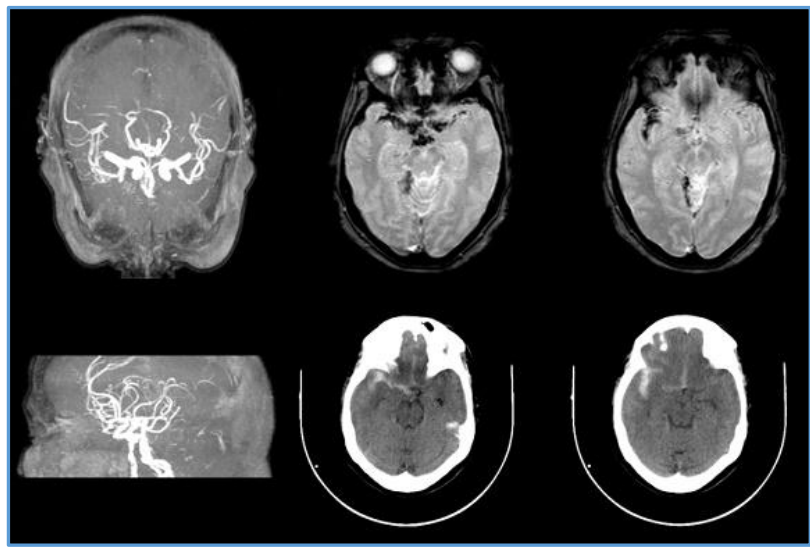

Figure 3. MR GRE Sequences and CT Brain plain images show sub arachnoid haemorrhage in right sylvian fissure, right basal cistern, interhemispheric fissure, inter peduncular cistern \& prepontine cistern. TOF MR Angiogram show narrow neck aneurysm at right MCA bifurcation

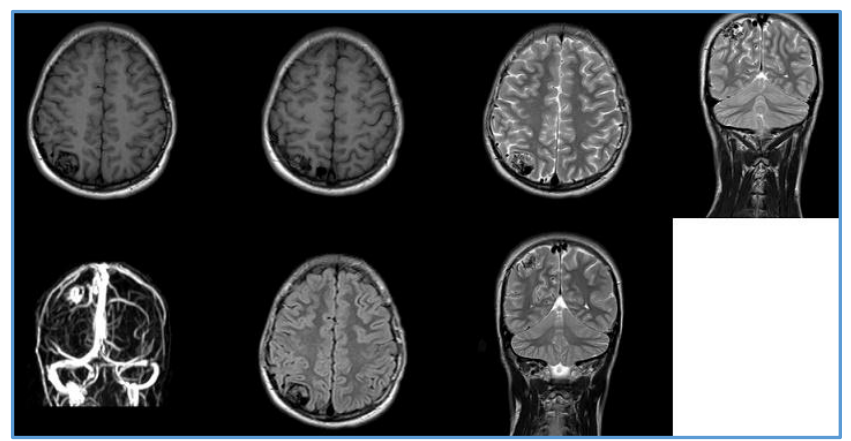

Figure 4. T1 \& T2 axial, T2 Sag \& coronal, TOF MR Angiogram \& FLAIR axial sequences show NIDUS in right parietal cortex. Feeding arteries are distal M4 branches of right MCA \& Draining into right parietal cortical veins and further to superior sagittal venous sinus---case of arterio venous malformation

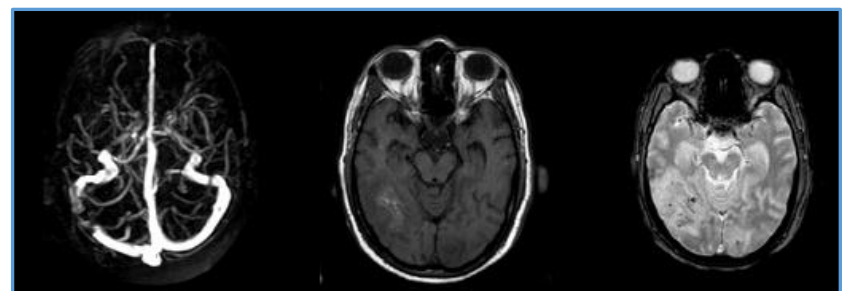

Figure 5. TOF MR Venogram, T1 \& GRE Sequences show Haemorrhagic infarcts in right parieto temporal lobe \& left occipital lobe with loss of flow signal in right transverse sinus \& adjacent right sigmoid sinus---case of venous sinus thrombosis

\section{DISCUSSION}

Intra cerebral haemorrhage is a major cause of morbidity and mortality. To identify the cause of haemorrhage is vital as the management and functional outcome depends upon the accurate diagnosis. The burden and distribution of cerebral micro haemorrhage has been correlated to patient outcome and is therefore an important marker of traumatic brain injury. 
Micro haemorrhages which are not evident on CT scan were identified on MRI especially in GRE and SWI sequences thus providing vital information in diagnosis of cases with diffuse axonal injury and amyloid angiopathy.

TOF MR Venogram provided vital information of venous sinus thrombosis in cases of venous infarcts compared to Plain CT scan.

In Cases of Arteriovenous malformations, compared to Plain CT scan, MRI T2, T1 and GRE sequences helped to better identify the nidus, feeding arterial branches and draining veins and venous sinuses.

\section{CONCLUSION}

In cases of intracerebral haemorrhage, MRI added crucial information to CT scan and aided in identifying the cause of intracerebral haemorrhage, diagnosis and management

\section{REFERENCES}

[1] Linn J. Imaging of cerebral microbleeds. Clin Neuroradiol 2015;25 Suppl 2:167-75.

[2] Sutton D. Textbook of radiology and imaging. $6^{\text {th }}$ edn. Edinburgh: Churchill Livingstone, 1998.

[3] Sussman ES, Connolly ES. Hemorrhagic transformation: a review of the rate of haemorrhage in the major clinical trials of acute ischemic stroke. Front Neurol 2013;4:69.

[4] Heit JJ, Michael Iv, Wintermark M, et al. Imaging of intracranial hemorrhage. J Stroke 2017;19(1):11-27. 